\title{
A Study of the evolution of microstructure and consolidation kinetics during sintering using a phase field modeling based approach
}

\author{
Sudipta Biswas ${ }^{\mathrm{a}}$, Daniel Schwen ${ }^{\mathrm{b}}$, Jogender Singh $^{\mathrm{c}}$ and Vikas Tomar ${ }^{\mathrm{a},{ }^{*}}$ \\ ${ }^{a}$ School of Aeronautics and Astronautics, Purdue University, West Lafayette, IN 47907, USA. \\ ${ }^{\mathrm{b}}$ Fuels Modeling and Simulation, IdahoNational Laboratory, Idaho Falls, ID 83415, USA \\ ${ }^{\mathrm{c}}$ Department of Material Science \& Engineering, Pennsylvania State University, University Park, PA \\ 16802, USA.
}

* Corresponding Author. Email: tomar@purdue.edu, Tel: 765-494-3423

\begin{abstract}
Sintering is a processing technique which compacts powder materials into solids leading to microstructural evolution with reduced surface area and improved material density. Understanding the densification mechanism and grain growth kinetics during the powder compaction process during sintering is of immense importance in order to evaluate usability of the final materials in a wide range of applications. Current work focuses on capturing the microstructural changes as powder particles compact into a polycrystalline structure during sintering. A phase field modeling based approach is adopted in this study in order to predict consolidation kinetics during sintering. It is observed that at the initial stage of sintering interactions between powder particles are initiated due to surface diffusion. At later stage, densification is primarily governed by volume and grain boundary diffusion. Also individual grains increase in size under pressure until adjacent grains touch each other. Once grains start interacting with adjacent grains, grain boundary diffuses and average grain size stabilizes.
\end{abstract}

Key Words: Sintering, Phase Field Modeling, Consolidation, Diffusion, Grain Growth 


\section{Introduction}

Sintering is an age old processing operation in powder metallurgy involving compaction of powder particles into a solid structure. It is a manufacturing technique which creates a solid polycrystalline structure from small powder particles with reduced surface area and improved density. Conventional solid state sintering methods focus on coalescing powder particles by applying heat and pressure without melting the powder material to the point of liquefaction. Solid state sintering in powder particles is activated due to mass diffusion or plastic flow or a combination of both. In the case of materials difficult to sinter using solid state sintering, liquid phase sintering is employed. In the case of liquid phase sintering external additives having melting points lower than the matrix powder material are added, so that they liquefy and act as an adhesive binding the powder particles together. With further developments electric, laser based, and microwave sintering processes have also emerged. A variant of spark plasma sintering (SPS) called as Field Assisted Sintering Technique (FAST) has been widely used and preferred over conventional sintering methods due to its significantly less processing time and its ability to perform sintering at lower process pressures. FAST also retains the sub-grain structure of the powder particles and prevents grain growth. FAST has been recently used in manufacturing of complicated structures involving joining of two different parts. The sintering based material processing also led to improvement in mechanical properties such as elastic modulus, hardness, fracture toughness etc. Sintering has been applied to processing of a variety of polycrystalline materials such as $\mathrm{Ni}, \mathrm{W}, \mathrm{Cu}$, Ti, Al, Mg, different ceramics etc. Quite a bit of work has been done in order to capture the sintering process through simulation [1-3], as well as experiments [4-6]. Theoretical models have been developed to identify the sintering mechanisms and establish constitutive relations. Temperature and pressure distribution across materials undergoing sintering have also been investigated [4]. Emphasis of the current work is on understanding interface evolution in powder particles during sintering. Importance is 
also given on aspects such as predicting the microstructural changes during and after sintering process. Tungsten is a candidate material for various high temperature applications due top it's high strength and low thermal conductivity. Sintering has been applied to compact tungsten powder for different applications. In order to validate the applicability of the model, tungsten is selected as material of choice for present analyses.

During sintering, consolidation or densification of powder particles takes place as powder particles unite into a solid material. Predicting the consolidation kinetics is a way to quantify evolution of microstructural features into powder material. Mostly consolidation kinetics has been explored for different materials using experiments [7-9]. Bruson [8] defined consolidation kinetics of an amorphous powder under warm pressing using effective material viscosity. During sintering the contraction between particles take place due to application of thermal and mechanical fluxes. Different resulting mass transport and diffusion mechanisms undergoing in powder material lead to accumulation of powder particles into larger grains [9]. It has been observed that depending on the external process condition and type of materials densification mechanisms change. When the applied loading is beyond yield strength of a material, plastic flow dominates the consolidation process, whereas for applied load being below yield strength densification occurs due to surface and grain boundary diffusion [10]. It has also been noticed that additives help in improving the rate of densification as well as the final density of the sintered material. With addition of $\mathrm{Cu}$, Ni or $\mathrm{HfC}$, the composite properties of $\mathrm{W}$ can be augmented [11]. In general, additive materials reduce the porosity of the raw material, resulting in enrichment of the density and mechanical properties. Different studies have been performed to observe how percentage addition of additives affect the powder consolidation $[12,13]$. Additionally, in other studies, effect of temperature, electricity and initial grain size has been considered separately [14-16]. Sintering, when done effectively with high load and for short time, can produce high density material from various 
powders limiting grain growth. Grigoryev [17] has shown how external process parameters such as electric discharge and pressure affects the densification kinetics. Experimental analysis demonstrated that with increase in electric discharge and external pressure, the rate of densification as well as final density of the material increases. However, there is an upper limit for both the parameters beyond which the powder disintegrates. Hu et. al. [18] have shown that lower heating rate results in higher relative density. However, higher heating rate yields higher rate of densification. At lower heating rate, the powder is exposed to elevated temperature for longer duration and hence, density of the material improves. It has also been observed [7] that different mechanical properties including the ultimate compressive strength of a material improves with sintering temperature. As of now the densification kinetics of a powder particles has been captured through experiments only. Significant amount of work has not been done to analytically relate consolidation mechanisms to microstructural changes during the compaction process.

Among the simulation work done till now, Monte Carlo simulation [19] and finite element modeling based approaches have dominated. Finite element modeling concentrates on capturing the sintering process considering the effect of external process parameters [4, 20-23] and Monte Carlo simulation relies on random sampling which is highly stochastic in nature. These approaches fail to correlate how the inherent microstructural features and properties affect the consolidation mechanisms during sintering. Hence, current work focuses on microstructural evolution of materials during the sintering process using the phase field modeling (PFM) approach. PFM is an effective tool for simulating microstructural evolution at meso-scale. In this method, microstructure is assumed to be a thermodynamically unstable structure which evolves in time and space for reaching equilibrium by minimizing the free energy. Microstructural features such as concentration of phases, grain orientation etc. are identified as phase field variables. The evolution of the phases, shape of the grains, positions of 
the interfaces are implicitly given by the evolution of the phase-field variables. It can consider several thermodynamic factors simultaneously, leading to microstructural changes such as bulk and interfacial energy, elastic energy, electric or magnetic energy etc. At the same time, it can take into account different transport mechanisms such as mass diffusion, heat conduction and convection [24]. For decades, phase field modelling has been applied to predict the impact of different material processing techniques including but not limited to diffusion [25], solidification[26-28], solid-state phase transformation [29, 30], translation of defects and dislocations [31-34], grain growth [35, 36], crack propagation $[37,38]$. During sintering, a material is subjected to different types of diffusion and mass transportation mechanisms, which can be well captured by PFM. All these factors make PFM the most viable option for analyzing the effect of sintering processes on the resulting material microstructure.

The objective of the current work is to analyze powder densification mechanisms and the consolidated powder microstructure during sintering. Primarily temporal evolution of the phase field variables, i.e., particle concentration is tracked to predict the consolidation kinetics. Neck formation and grain growth during sintering is evaluated to comprehend the consolidation mechanisms. Impact of initial grain size on densification and grain growth is captured.

\section{Phase Field Model Formulation}

PFM is a thermodynamic modeling approach for capturing microstructural evolution under various thermo-mechanical boundary conditions. This process assigns phase field variables to all the constituent phases and microstructural features of the analyzed material. The aim is to capture the numerical changes in assigned variables which directly signify the alteration in material microstructure. The equations for the evolution of phase-field variables are derived based on general thermodynamic principles [24]. The driving force for microstructural evolution in PFM is a prospect to reduce the free energy of the analyzed system. The total free energy $F$ in PFM is formulated as a function of the phase- 
field variables and order parameters, which are functions of time and spatial coordinates. Total free energy can be represented as,

$$
F=\int\left[f_{0}(c, \eta)+f_{e l}(c, \eta)+\frac{\kappa_{c}}{2}|\nabla c|^{2}+\frac{\kappa_{\eta}}{2}|\nabla \eta|^{2}\right] d V
$$

where $f_{0}(c, \eta)$ is the bulk free energy of the material which determines the volume fraction of the coexisting phases at equilibrium condition, $f_{e l}(c, \eta)$ is the elastic energy contribution, $\kappa_{c}$ and $\kappa_{\eta}$ are gradient co-efficients, $c$ is the conserved phase field variable representing the particle concentration, and $\eta$ is an nonconserved order parameter [24] which varies with space and time. The $2^{\text {nd }}$ and the $3^{\text {rd }}$ term in Eq. (1) denote the gradient free energy from the surface and grain boundaries, respectively. Gradient free energy determines the interface evolution during particle interactions and dictates changes in the shape of the particles during the material processing. Elastic energy coupling enables consideration of mechanical loading along with PFM.

In PFM, the temporal evolution of the phase-field variables is given by a set of coupled partial differential equations, one equation for each variable. The equations are derived according to the principles of non-equilibrium thermodynamics. They are formulated in such a way that the total free energy decreases monotonically and mass is conserved for all components. Solution of the partial differential equations yields the temporal and spatial evolution of the phase-field variables, which is a representation of the total morphological evolution of the material microstructure.

\subsection{Chemical Free Energy:}

Bulk free energy is the main component of the free energy potential under consideration for PFM. This is the chemical free energy inherited by processed material due to its constituent phases. This can be obtained from molecular dynamics or quantum simulations. CALPHAD can also be used to derive 
the chemical free energy of a multi-phase system. In the current work, the basic chemical free energy has been taken from Deng [39]. Chemical free energy is represented as,

$$
f_{0}\left(c, \eta_{i}\right)=A c^{2}(1-c)^{2}+B\left[c^{2}+6(1-c) \sum_{i} \eta_{i}^{2}-4(2-c) \sum_{i} \eta_{i}^{3}+3\left(\sum_{i} \eta_{i}^{2}\right)^{2}\right],
$$

where, $c$ is particle concentration in the material, $\eta$ is crystallographic orientation of particles, $A$ and $B$ are constants material parameter which depends on grain boundary and surface energies.

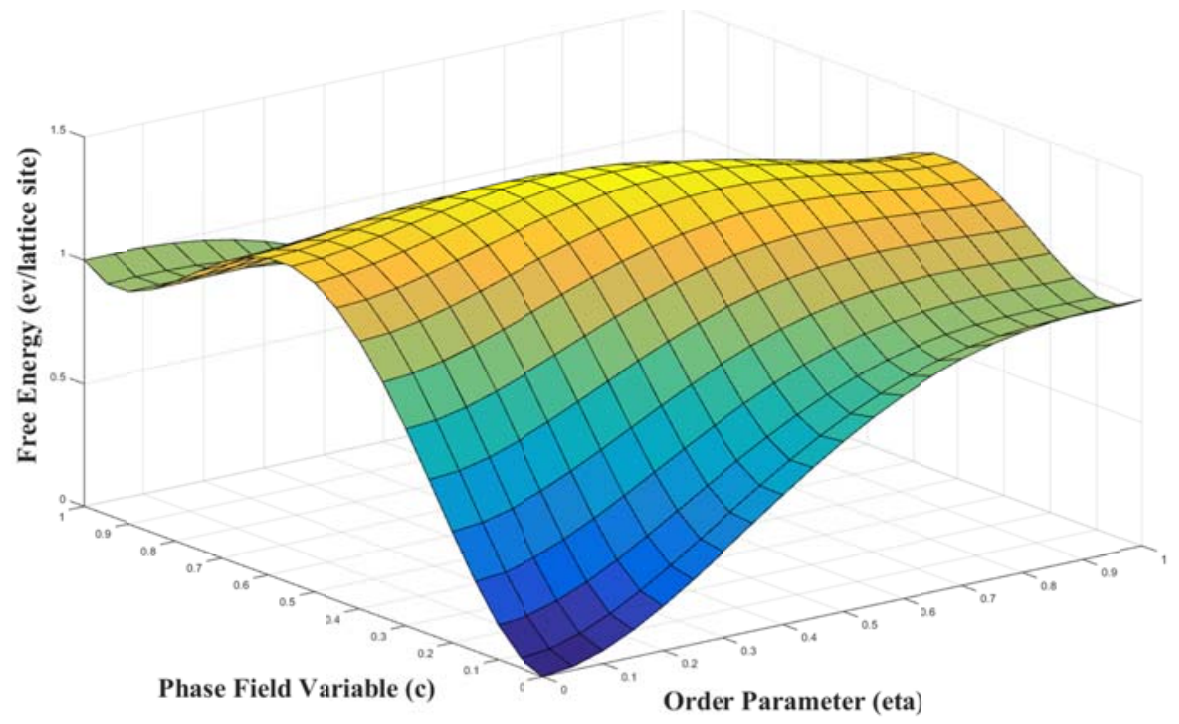

\section{Figure 1. Chemical free energy as a function of particle concentration and order parameter}

Fig. (1) plots the chemical free energy used in the simulations. As observed in the figure, bulk free energy is minimum at each of the pure particle or void phases, whereas being maximum at the interface. The energy formulation is constructed in a way that particle concentration $\mathrm{c}=0$ in voids and $\mathrm{c}=1$ in solid particles. The equilibrium value of the multicomponent order parameter is reached in solid with $\{\eta\}_{\rho=1}=\{1,0,0 \ldots \ldots . .0\}=\{0,1,0 \ldots \ldots . .0\}=\ldots \ldots \ldots \ldots . .=\{0,0,0 \ldots \ldots .1\} \quad$ and $\quad$ vanishes $\quad$ in pores, i.e. 
$\{\eta\}_{\rho=0}=\{0,0,0 \ldots \ldots . .0\}$. The interface evolves to minimize the free energy leading to evolution of the microstructure.

\subsection{Elastic Energy Contribution:}

During phase transformation, in the case of solids, elastic stresses are induced into the material owing to lattice mismatch between grains or externally applied loads. The elastic properties of different co-existent phases are different. And, hence, the stresses experienced by the phases are different leading to different elastic energy contribution. Further deformation leads to plastic energy and dissipation, which is a subject of future study. First, depending on the applied load elastic strains are calculated. The elastic strain, here, is defined as the difference between the total strain and the stress free strain,

$$
\varepsilon_{i j}^{e l}=\varepsilon_{i j}^{t o t a l}-\varepsilon_{i j}^{0}
$$

Stress free strain corresponds to Eigenstrain due to lattice mismatch, residual stresses after manufacturing and thermal strain. For the current case, it is assumed that no stress free strain exists between powder particles and voids during initial powder stacking stage $\left(\varepsilon_{i j}^{0}=0\right)$. From elastic strain elastic stresses as computed using Hook's law as,

$$
\vec{\sigma}_{i j}(r)=C_{i j k l}(\vec{r}) \varepsilon_{k l}^{e l}(\vec{r})
$$

where, $C_{i j k l}(\vec{r})$ are the components of the elastic modulus tensor which are unique for particle and void phases and vary with concentration. Considering the fact that mechanical equilibrium is obtained much before the chemical equilibrium, strain is obtained by solving the equilibrium equation,

$$
\frac{\partial \sigma_{i j}}{\partial r_{j}}=0
$$


In order to simplify the solution, total stress is assumed to consist of a homogeneous macroscopic strain and a heterogeneous local strain components,

$$
\varepsilon_{i j}^{t o t a l}=\bar{\varepsilon}_{i j}+\delta \varepsilon_{i j}(r)
$$

Here, the homogeneous strain is such that,

$$
\int_{V} \delta \varepsilon_{i j}(r) d^{3} r=0
$$

and the heterogeneous component can be computed from the local displacements as,

$$
\delta \varepsilon_{k l}(r)=\frac{1}{2}\left[\frac{\partial u_{k}(r)}{\partial r_{l}}+\frac{\partial u_{l}(r)}{\partial r_{k}}\right]
$$

Here, $u_{i}$ is the component of the displacement vector $\vec{u}$. Solid mechanics in a fully tensorial form is utilized for calculating the displacement and corresponding strains. After obtaining the strains, the elastic energy is computed as,

$$
f_{e l}=\frac{1}{2} \int_{V} C_{i j k l}(\vec{r}) \varepsilon_{i j}^{e l} \varepsilon_{k l}^{e l} d \vec{r}
$$

When the effect of the external forces are also taken into consideration, the strain energy expression becomes,

$$
f_{e l}=\frac{1}{2} \int_{V} C_{i j k l}(\vec{r}) \varepsilon_{i j}^{e l} \varepsilon_{k l}^{e l} d \vec{r}-\int_{V} \sigma_{i j}^{a}(\vec{r}) \varepsilon_{i j}^{e l} d \vec{r}
$$

The elastic strain energy is accounted into the total free energy equation of the phase field model and the combined effect of the external load along with the other process parameters can be examined. Since elastic properties vary with phase concentration, stresses in each phase are also different. Global stress is calculated using following equation, 


$$
\sigma_{\text {global }}=h(c) \sigma_{\text {particle }}+(1-h(c)) \sigma_{\text {void }}
$$

Here, $h(c)$ is the switching function such that $h=3 c^{2}-2 c^{3}$. In this work, the elastic properties used in the elastic energy formulation are tabulated in Table 1.

Table 1. Elastic Properties of W [40]

\begin{tabular}{|c|c|}
\hline Young's modulus, $\mathbf{E}$ & $390 \mathrm{GPa}$ \\
\hline Poisson's ratio, $\mathbf{v}$ & 0.27 \\
\hline Maximum principal stress, $\mathbf{T}$ & $4000 \mathrm{MPa}$ \\
\hline
\end{tabular}

\subsection{Particle Rigid Body Motion:}

During sintering the whole powder particles can translate and rotate on top of each other. The rigid body motion of particles during sintering has been implemented in current model using advection velocities of individual grains. In this regard first the force density acting on each grain has been computed using,

$$
d F_{i}=k \sum_{j \neq i}\left(c-c_{0}\right)\left\langle\eta_{i} \eta_{j}\right\rangle\left[\nabla \eta_{i}-\nabla \eta_{j}\right] d^{3} r
$$

where, $k$ is stiffness constant relating to force magnitude due to mass density variation at grain boundaries and $\rho_{0}$ is the equilibrium density. $\left\langle\eta_{i} \eta_{j}\right\rangle$ is defined as,

$$
\left\langle\eta_{i} \eta_{j}\right\rangle=\left\{\begin{array}{ll}
1, & \text { for } \eta_{i} \eta_{j} \geq c_{g b} \\
0, & \text { otherwise }
\end{array} .\right.
$$

Parameter $c_{g b}$ is the threshold concentration value for grain boundaries. The force density corresponds to the reaction forces between two particles as they interact with each other during the compaction 
process. The volumes and centers of each grain has been calculated so that the total force and torque acting on each grain can be estimated. Here, grain center $\left(\mathrm{r}_{\mathrm{ci}}\right)$ is the center of mass of the $i^{\text {th }}$ particle,

$$
r_{c i}=\frac{1}{V_{i}} \int_{V} r \eta_{i} d^{3} r \quad \text { with } \quad V_{i}=\int_{V} \eta_{i} d^{3} r
$$

where, $V_{i}$ is the volume of the $i^{\text {th }}$ grain. Similarly, the forces $\left(F_{i}\right)$ and torques $\left(T_{i}\right)$ acting on the particles are calculated as,

$$
\begin{gathered}
F_{i}=\int_{V} d F_{i}, \text { and } \\
T_{i}=\int_{V}\left[r-r_{c i}\right] \times d F_{i}
\end{gathered}
$$

with $\mathrm{r}$ being the position vector. Finally, the grain advection velocity corresponding to each grain is calculated as,

$$
\begin{gathered}
v_{t i}=\frac{m_{t}}{V_{i}} F_{i} \eta_{i}, \\
v_{r i}=\frac{m_{r}}{V_{i}} T_{i} \times\left[r-r_{c i}\right] \eta_{i}, \text { and } \\
v_{a d v_{i}}=v_{t i}+v_{r i} .
\end{gathered}
$$

Here, $v_{\mathrm{ti}}$ and $v_{\mathrm{ri}}$ are the translational and rotational velocities of grains and $\mathrm{m}_{\mathrm{t}} \& \mathrm{~m}_{\mathrm{r}}$, are the translational $\&$ rotational masses, respectively. Total advection velocity of a grain $\left(v_{\text {advi }}\right)$ is a combination of translational and rotational velocities. Advection velocity terms are used to modify the traditional phase field equations to implement rigid body motion of the particles. This model has been adopted from 
Wang [41]. The method of coupling rigid body motion with phase-field will be discussed in details in the following numerical implementation section.

\subsection{Numerical Implementation:}

A numerical formulation is developed to capture the spatial and temporal evolution of the phase field variables. Cahn-Hilliard $(\mathrm{CH})$ equation [42] is used to track the conserved variable as,

$$
\frac{\partial c}{\partial t}(\vec{r}, t)=\nabla \cdot\left[M \nabla \frac{\delta F}{\delta c}\right]
$$

Similarly, the evolution of the order parameter is defined using the Allen-Cahn (AC) equation as,

$$
\frac{\partial \eta_{i}}{\partial t}(\vec{r}, t)=-L \frac{\delta F}{\delta \eta_{i}}
$$

where, $F$ is the total free energy of the system as defined in Eq. (13) earlier, parameter $\mathrm{M}$ is the diffusion mobility as defined in Section 2.5 and parameter $L$ is the grain boundary mobility due to atomic jump across grain boundary or due to grain boundary transition. In order to incorporate the rigid body motion conventional $\mathrm{CH}$ and $\mathrm{AC}$ equations are modified as follows,

$$
\begin{gathered}
\frac{\partial c}{\partial t}(\vec{r}, t)=\nabla \cdot\left[M \nabla \frac{\delta F}{\delta c}-c \sum_{i} v_{a d v_{i}}\right] \\
\frac{\partial \eta_{i}}{\partial t}(\vec{r}, t)=-L \frac{\delta F}{\delta \eta_{i}}-\nabla \cdot \eta_{i} v_{a d v_{i}}
\end{gathered}
$$

The modified equations incorporates grain advection terms for capturing rigid body motion of particles. This term is effective only when particles are off-set from each other. $v_{a d v_{i}}$ is the advection velocity of the $i^{\text {th }}$ particle.

\subsection{Mobility Calculation:}

Mobility corresponding to $\mathrm{CH}$ equation, calculated from the diffusion coefficiens, is defined as, 


$$
M=M_{v o l} \phi(c)+M_{v a p}[1-\phi(c)]+M_{\text {surf }} c(1-c)+M_{g b} \sum \eta_{i} \eta_{j},
$$

where $\phi(c)=c^{3}\left(10-15 c+6 c^{2}\right)$ and $M_{v o l}, M_{v a p} M_{\text {surf }}, M_{g b}$ are the coefficients for atomic mobility in solid bulk, vapor, along surface and grain boundary phases, [41]. Mobility co-efficients $\left(M_{i}\right)$ are estimated, according to the work of Moelens et al. [24], from corresponding diffusion co-efficients as,

$$
M_{i}=\frac{D_{i} V_{m}}{k_{B} T}
$$

Here, D is the diffusion co-efficients and suffix $i$ corresponds to volme, vapor, GB and surface diffusion; $V_{m}$ is the molar volume, $k_{B}$ is boltzman constant and $T$ is the absolute temperature. The diffusion coefficients can be expressed as a function of temperature $(T)$ and activation energy $\left(Q_{i}\right)$ values as,

$$
\begin{gathered}
D_{G B}=D_{G B 0} \exp \left(-Q_{G B} / k_{B} T\right), \\
D_{b u l k}=D_{0} \exp \left(-Q / k_{B} T\right), \text { and } \\
D_{s}=D_{s 0} \exp \left(-Q_{s} / k_{B} T\right) .
\end{gathered}
$$

Here, $D_{i 0}$ are the diffusion prefactors used for the diffusivity calculation. Prefactors and the activation energies used in mobility calculation are extracted from the literature [43-45]. The gradient energy coefficients i.e. kappa values are calculated based on the grain boundary and surface energy values of the materials as,

$$
\begin{gathered}
\kappa_{c}=k_{1}\left(2 \gamma_{s}-\gamma_{G B}\right) * \delta \text {, and } \\
\kappa_{\eta}=k_{2}\left(\gamma_{G B}\right) * \delta .
\end{gathered}
$$

The constants $A \& B$ in the free energy formulation are obtained as, 


$$
\begin{gathered}
A=k_{3}\left(12 \gamma_{s}-7 \gamma_{G B}\right) / \delta \text {, and } \\
B=k_{4}\left(\gamma_{G B}\right) / \delta .
\end{gathered}
$$

$\gamma_{s}$ and $\gamma_{G B}$ are the surface and grain boundary energy, respectively. $\delta$ is the interface width also considered as the grain boundary thickness. $k_{1}, k_{2}, k_{3}, k_{4}$ are the constants used for normalizing the material parameters. Above correlations in Eqs. (29) to (32) are adopted from the work of El-Azab et. al [46], where a similar model is used for observing grain growth in a porous solid. The co-efficients have been modified according to the current simulation requirements. Modeling sintering is different from generic grain growth models in a way that it includes external loading and rigid body movement of the particles, combining both together for capturing the sintering mechanisms is the original contribution of this work. A parametric study has been executed to observe how different energy and mobility ratio effects the densification and grain growth model. Calculation of the free energy constants and gradient energy co-efficients are provided in Table (2).

\begin{tabular}{|c|c|c|c|c|c|}
\hline $\begin{array}{l}\text { GB Energy } \\
\left(\mathrm{ev} / \mathrm{nm}^{2}\right) \\
{[47]}\end{array}$ & $\begin{array}{l}\text { Surface Energy } \\
\left(\mathrm{ev} / \mathrm{nm}^{2}\right) \\
{[47]}\end{array}$ & $\begin{array}{l}\text { A } \\
\left(\mathrm{ev} / \mu \mathrm{m}^{3}\right)\end{array}$ & $\begin{array}{l}\text { B } \\
\left(e v / \mu m^{3}\right)\end{array}$ & $\begin{array}{l}\kappa_{\mathrm{c}} \\
(\mathrm{ev} / \mu \mathrm{m})\end{array}$ & $\begin{array}{l}\kappa_{\eta} \\
(e v / \mu m)\end{array}$ \\
\hline 42.85 & 58.23 & $1.33 \times 10^{-11}$ & $3.43 \times 10^{-12}$ & $8.36 \times 10^{-12}$ & $1.03 \times 10^{-11}$ \\
\hline
\end{tabular}

Table 2. Energy co-efficients calculated for Tungsten

Table (3) shows the calculated mobilities for tungsten at $1200 \mathrm{~K}$ temperature.

Table 3. Mobility Calculation for Tungsten 


\begin{tabular}{|c|c|c|c|c|c|c|c|c|}
\hline $\begin{array}{l}\text { Diffusion } \\
\text { prefactor } \\
\left(\mathrm{cm} / \mathrm{s}^{2}\right)\end{array}$ & $\begin{array}{l}\text { Activation } \\
\text { energy } \\
\text { (ev) }\end{array}$ & $\begin{array}{l}\text { Mobility } \\
\left(\mu \mathrm{m}^{5} / \mathrm{ev} \mathrm{s}\right)\end{array}$ & $\begin{array}{l}\text { Diffusion } \\
\text { prefactor } \\
\left(\mathrm{cm} / \mathrm{s}^{2}\right)\end{array}$ & $\begin{array}{l}\text { Activation } \\
\text { energy } \\
\text { (ev) }\end{array}$ & $\begin{array}{l}\text { Mobility } \\
\left(\mu \mathrm{m}^{5} / \mathrm{evs}\right)\end{array}$ & $\begin{array}{l}\text { Diffusion } \\
\text { prefactor } \\
\left(\mathrm{cm} / \mathrm{s}^{2}\right)\end{array}$ & $\begin{array}{l}\text { Activation } \\
\text { energy } \\
\text { (ev) }\end{array}$ & $\begin{array}{l}\text { Mobility } \\
\left(\mu \mathrm{m}^{5} / \mathrm{evs}\right)\end{array}$ \\
\hline 0.141 & 3.04 & $3.69 \times 10^{15}$ & 4.0 & 3.14 & $3.98 \times 10^{15}$ & 0.04 & 5.4 & $1.295 \times 10^{4}$ \\
\hline
\end{tabular}

Mobility co-efficients $M_{g b}, M_{v o l}, M_{\text {surf }}$ have been calculated separately and fitted into Eq. (24). For avoiding numerical issues and facilitate fast convergence, all the simulation parameters have been normalized with respect to the grain boundary energy. Also appropriate length and time scale has been used to nondimesionalize the simulation. Changes in the energy ratio impact the interfacial and material parameters, which eventually affect the microstructural evolution. Hence, energy ratio and corresponding interfacial parameters have been varied to observe the effect of energy ratio on the neck formation and grain growth during sintering. Nondimensionalized parameters used for the study are summarized in Table. (4).

Table 4. Nondimensionalized Simulation Parameters used in Parametric Study

\begin{tabular}{|l|l|l|l|l|l|l|l|l|l|}
\hline Parameters & $\boldsymbol{\kappa}_{\boldsymbol{\eta}}$ & $\boldsymbol{\kappa}_{\mathbf{c}}$ & $\mathbf{A}$ & $\mathbf{B}$ & $\mathbf{M}_{\mathbf{v o l}}$ & $\mathbf{M}_{\mathbf{v a p}}$ & $\mathbf{M}_{\text {surf }}$ & $\mathbf{M}_{\mathbf{g b}}$ & $\mathbf{L}$ \\
\hline Case I & 0.5 & 1.0 & 16.0 & 1.0 & 0.0129 & 0.001 & 3.98 & 0.37 & $\mathrm{M}$ \\
\hline Case II [41] & 1.0 & 10.0 & 16.0 & 1.0 & 0.01 & 0.001 & 4.0 & 0.4 & $10 \mathrm{M}$ \\
\hline Case III & 0.5 & 1.0 & 2.6 & 1.5 & 0.0129 & 0.001 & 3.98 & 0.37 & $\mathrm{M}$ \\
\hline
\end{tabular}

\subsection{Modeling Approach and Simulation Tool:}

In this work, we have used Idaho National Lab's (INL's) Multiphysics Object-Oriented Simulation Environment (MOOSE) for implementing the current phase field model and extending it's 
coupling with solid/tensor mechanics. MOOSE is a massively parallel system for solving sets of differential equations via the finite element method (FEM). It provides an implicit framework for modeling and solving differential equations corresponding to a variety of physics modules. MOOSE emphasizes on spending less development time by maximizing code re-use. It has in-built classes for modelling the basic $\mathrm{CH}$ and $\mathrm{AC}$ phase field equations. Only the free energy terms and the material models need to be taken care of. It also has the capability of multiphysics coupling such as combining phase field with solid mechanics, heat conduction etc. This makes incorporating effects of external elastic loading straight forward for the current problem. All these features motivated us to choose MOOSE framework as the simulation tool.

Numerical solution of Eq. (22) gives the temporal evolution of the phase-field variable which represents the changes in density of the system. For finite element formulation, Eqs. (22) and (23) are further derived into weak forms for implementation. In order to develop the weak form, first the strong form equation is expressed in terms of all the energy contributions. The $\mathrm{CH}$ equation can be rewritten as,

$$
\frac{\partial c}{\partial t}=\nabla \cdot M \nabla\left(\frac{\delta f_{0}}{\delta c}+\frac{\delta f_{d}}{\delta c}-k_{c} \nabla^{2} c\right)-\nabla \cdot\left(c \sum_{i} v_{a d v_{i}}\right)
$$

Eq. (33), here, is a $4^{\text {th }}$ order PDE which requires higher order elements to be solved using the finite element method. There are two ways this equation can be solved. One being the direct solution of the equation by deriving the weak form using higher order elements, whereas the other method splits this equation into two $2^{\text {nd }}$ order equations and uses linear elements to solve them. Zhang et. al [48] has shown that both methods work efficiently and are giving comparable results. However, the $2^{\text {nd }}$ method involves faster convergence, lesser computational time with a very minimal error due to use of linear Lagrange elements. For current work the $2^{\text {nd }}$ method using a split $\mathrm{CH}$ equation has been adopted. The strong form of the split version of $\mathrm{CH}$ equation is expressed as, 


$$
\begin{gathered}
\frac{\partial c}{\partial t}=\nabla \cdot\left(M \nabla \mu-c \sum_{i} v_{a d v_{i}}\right), \text { and } \\
\mu=\frac{\delta f_{0}}{\delta c}+\frac{\delta f_{d}}{\delta c}-k_{c} \nabla^{2} c .
\end{gathered}
$$

Deriving weak form for finite element discretization using same scheme as Tonks et.al. [49], Eqs. (34) and (35) yield,

$$
\begin{gathered}
\left(\frac{\partial c}{\partial t}, \psi_{m}\right)=\left(M \nabla \mu, \nabla \psi_{m}\right)-\left(\nabla \cdot\left(c \sum_{i} v_{a d v_{i}}\right), \psi_{m}\right)-\left\langle M \nabla \mu \cdot \vec{n}, \psi_{m}\right\rangle, \text { and } \\
\left(\mu, \psi_{m}\right)=\left(\frac{\delta f_{0}}{\delta c}+\frac{\delta f_{d}}{\delta c}, \psi_{m}\right)+\left(k_{c} \nabla c, \nabla \psi_{m}\right)-\left\langle k_{c} \nabla c \cdot \vec{n}, \psi_{m}\right\rangle .
\end{gathered}
$$

$\psi_{m}$, here, is the finite element test function. Weak form equations are completed with initial and boundary conditions. Usual inner product notation has been used for volume and boundary integrals. Similarly, AC equation can be expressed in strong form as,

$$
\frac{\partial \eta_{i}}{\partial t}=-L\left(\frac{\delta f_{0}}{\delta \eta_{i}}+\frac{\delta f_{d}}{\delta \eta_{i}}-k_{\eta_{i}} \nabla^{2} \eta_{i}\right)-\nabla \cdot\left(\eta_{i} v_{a d v_{i}}\right)
$$

Further, the weak form of Eq. (38) is,

$$
\left(\frac{\partial \eta_{i}}{\partial t}, \psi_{m}\right)=-L\left(\frac{\delta f_{0}}{\delta \eta_{i}}+\frac{\delta f_{d}}{\delta \eta_{i}}, \psi_{m}\right)+L\left(k_{\eta_{i}} \nabla \eta_{i}, \nabla \psi_{m}\right)-\left(\nabla \cdot \eta_{i} v_{a d v_{i}}, \psi_{m}\right)-L\left\langle k_{\eta_{i}} \nabla \eta_{i} \cdot \vec{n}, \psi_{m}\right\rangle .
$$

Details of the mathematical modeling and numerical scheme for solving these equation have been provided by Tonks et. al. [23]. Fig. (2) shows the rationale behind the modeling approach along with the boundary condition of the model. Sintering involves application of pressure and temperature in an 
enclosed volume. For the initial part we start with two circular particles in 2D and thereafter, focused on multiple particles.

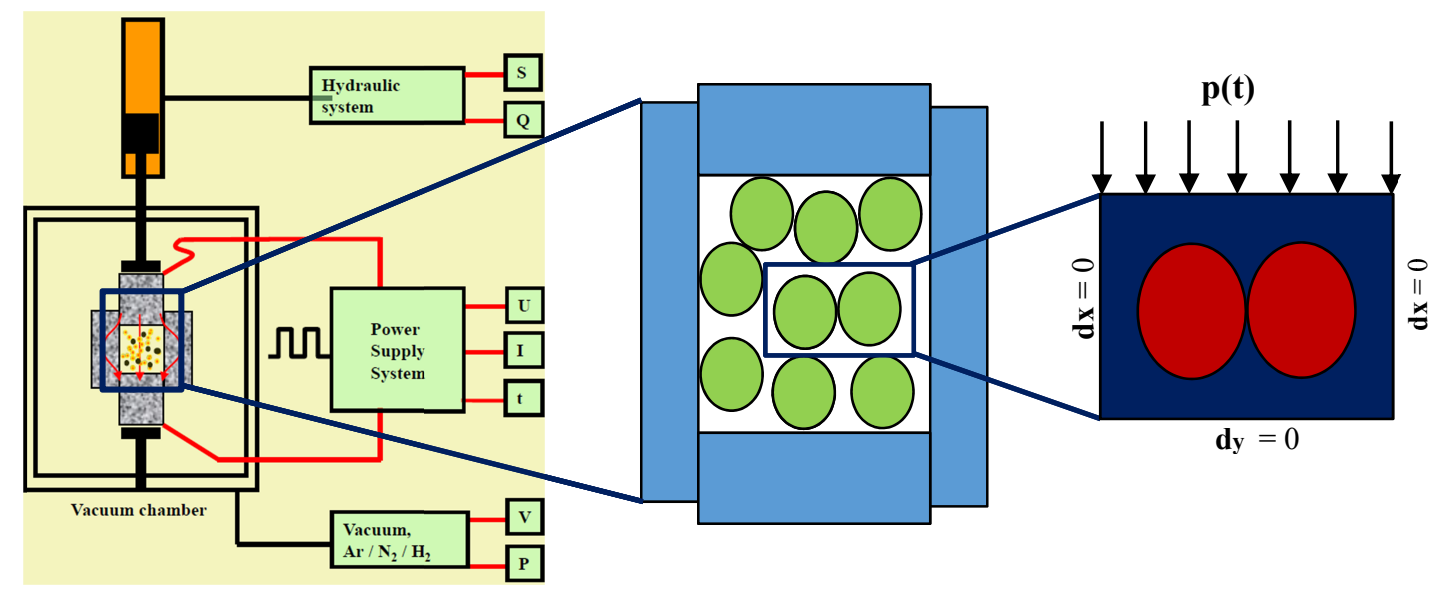

(a)

(b)

(c)

Figure 2. Modelling Approach: Circular particles in 2D; (a) Schematic of a sintering process;

(b) Schematic of the sintering mold; (c) Simplified geometry adoped in modeling.

We consider part of the microstructure as our simulation domain. The initial domain has been chosen to be $20 \mu \mathrm{m} \mathrm{X} 40 \mu \mathrm{m}$. Initial values of the phase filed variables are assigned as described in section 2.1 and the evolution of microstructure is studied to get the consolidation kinetics of powder. Periodic boundary conditions have been used for all phase field variables, i.e., for concentration field and order parameters. The displacement of the bottom edge has been constrained in the y direction and the sides are restricted to move in $\mathrm{x}$ direction. In this case, particles are assumed to be in contact at initial stage.

\section{Results:}

For the preliminary verification, a two particle model with two same size circular particles has been studied for neck growth during sintering. Contribution of both the chemical free energy and elastic energy is taken into account. In this case, it is observed that the chemical potential of the material governs the neck growth phenomenon. External load, here, contributes to the shape change of the 
paricles during compaction. Fig. (3) shows concentration variations leading to the microstructural changes and neck growth during sintering.

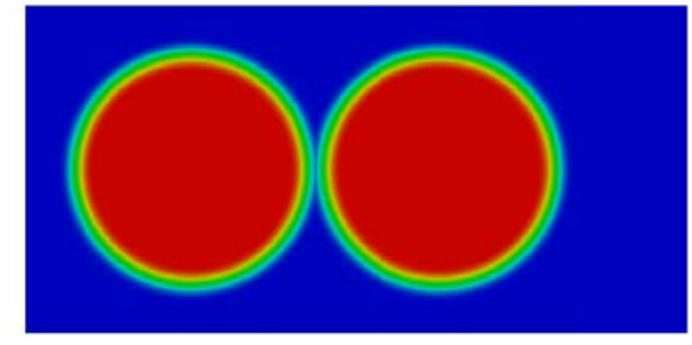

(a)

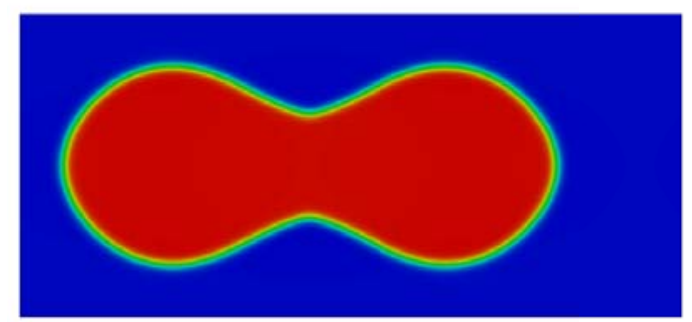

(c)

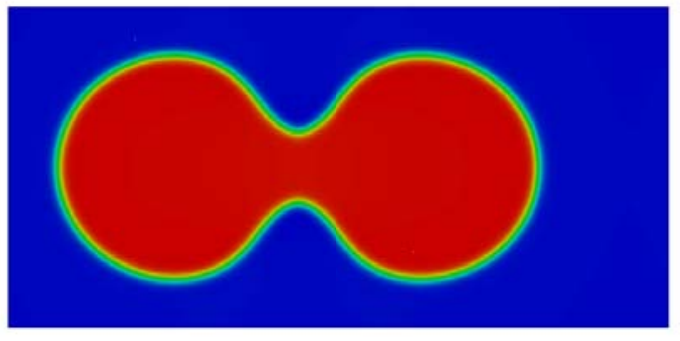

(b)

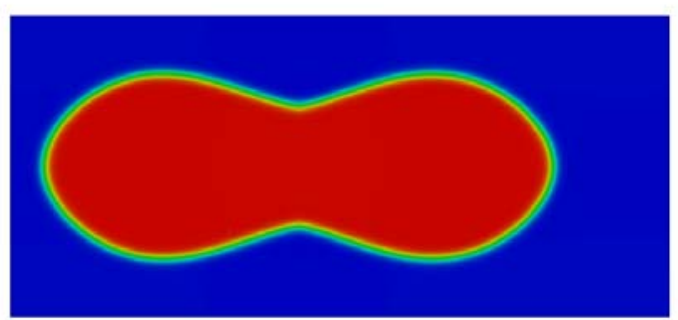

(d)

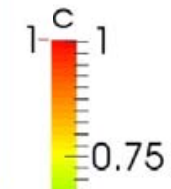

0.5

0.25

Figure 3. Concentration variation for model with two particle of same size at a) 0 sec b) 10 sec c) $50 \mathrm{sec} d) 100 \mathrm{sec}$

Similarly, variation of order parameter is shown in Fig. (4).

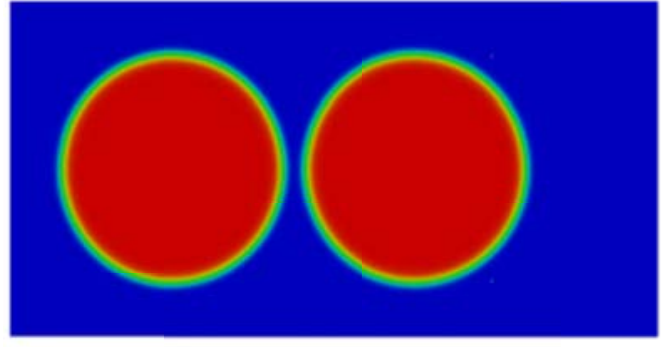

(a)

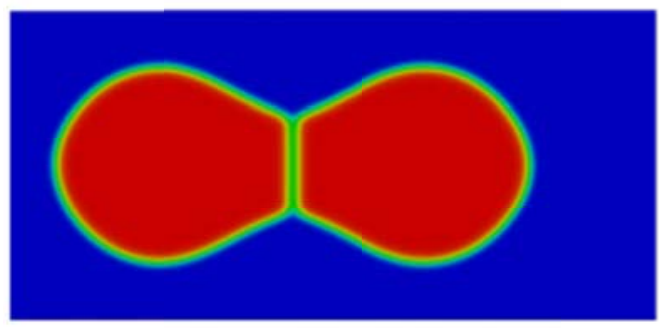

(c)

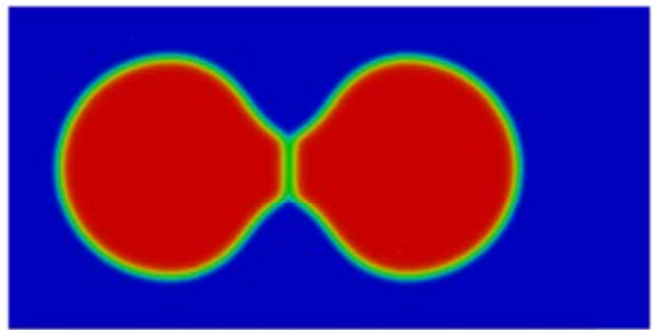

(b)

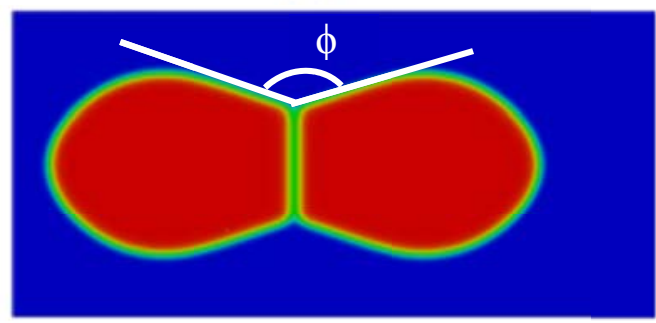

(d)

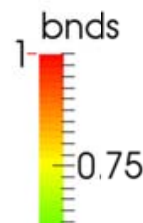

0.5 
Figure 4. Order parameter variation (representing grain orientations) for model with two particle of same size at a) $0.1 \mathrm{sec}$ b) $10 \mathrm{sec}$ c) $50 \mathrm{sec}$ d) $100 \mathrm{sec}$

The red zone in Fig. (3) \& (4) represents the parlicle concentration and orientation, respectively. The blue zone is void in both the cases and the concentration varies over the interface between particle and void. Fig. 4 shows the formation of grain boundaries and additional variable ' $b n d s$ ' has been introducesd for plotting purpose. 'Bnds' is a combination of all the order parameters and is defined as, bnds $=\sum \eta_{i} \eta_{j}$ with $\eta$ being the grain orientation of the partciles. Formation of a grain boundary between two particles is evident when microstructural evolution is observed in terms of order parameter variation. Fig. 4(d) also clearly shows the dihedral angle $(\varphi)$ formed between two particles. This dihedral angle varies depending on grain boundary to surface energy ratio, mobility and interfacial parameters. The equilibrium dihedral angle is determined as;

$$
\varphi=2 \cos ^{-1}\left(\frac{\gamma_{G B}}{2 \gamma_{s}}\right)
$$

where, $\varphi$ is the dihedral angle between grains at the neck location and $\gamma_{G B} \& \gamma_{s}$ are the grain boundary \& surface energies, respectively. Different energy ratio yields different equilibrium dihedral angles. For further validation, density evolution along the neck line and neck growth over time has been studied. Fig. (5a) tracks the change in density along neck line and Fig. (5b) represents the neck growth over time. 


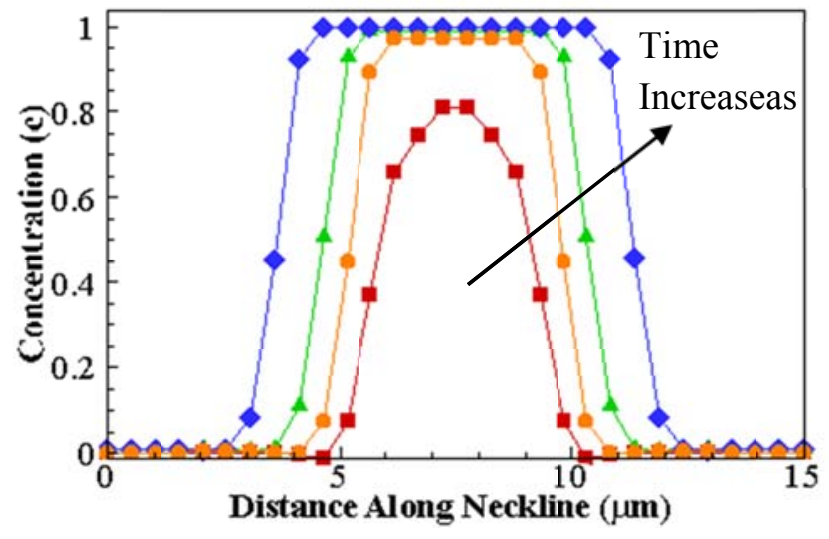

(a)

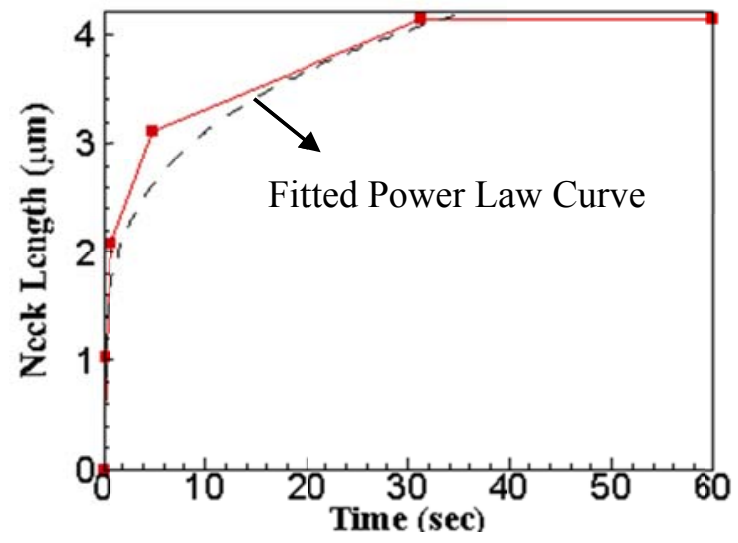

(b)

Figure 5. a) Density variation along neck line at different time steps, b) Change in neck length over time

As observed in Fig. (5a) two particles are just in contact at time zero and as time progresses two particles interact with each other and contact area between particles increases i.e. neck formation starts. Similarly, also from Fig. (5b) it is noticeable that neck size increases with time. However, the neck growth rate is high at initial times and it flattens over period of time. During initial stage of sintering the curvature of the particle surfaces at the neck location is high and this raises the driving force for neck growth. As sintering progresses, the surface becomes smoother and neck growth rate decreases. Curve fitting of the data shows that neck growth follows the following relation,

$$
\log (D)=C_{1} \log (t)+C_{2}
$$

where $C_{1}, C_{2}$ are constants obtained as 0.24 and 1.05 for this case. Previously, Wang [41] and Deng [39] have shown that neck growth follows power law such as $(x / D)^{n} \propto t$ where $x$ is the neck length, $D$ is the domain length and $t$ is sintering time. Relations by both researchers are comparable and predict similar results. Following the power law, the exponent obtained in this case would be approximately 4.2 which is in agreement with values found by Deng [39]. The exponent value obtained here is different from the 
theoretical values since sintering is governed by mixed diffusion mechanisms. During initial stage because of higher curvature, neck formation starts due to surface diffusion. At later stages densification is governed by volume and grain boundary diffusion. Also the effect of the surface energy to grain boundary energy ratio on the neck length and sintering mechanism, has been studied. Fig. (6) shows neck length variation corresponding to three different energy ratio values. The energy ratio $\left(\gamma_{\mathrm{s}} / \gamma_{\mathrm{gb}}\right)$ is controlled by changing the ratio of $\kappa_{\mathrm{c}} \& \kappa_{\eta}$ and primarily effects the initial sintering stage when neck formation is dominated by surface diffusion. Higher energy ratios indicate higher surface energies and higher $\kappa_{\mathrm{c}}$ values, which leads to faster diffusion at initial stage. The rate of densification increases with increase in energy ratio. Also the time to reach final neck length decreases with incease in energy ratio.

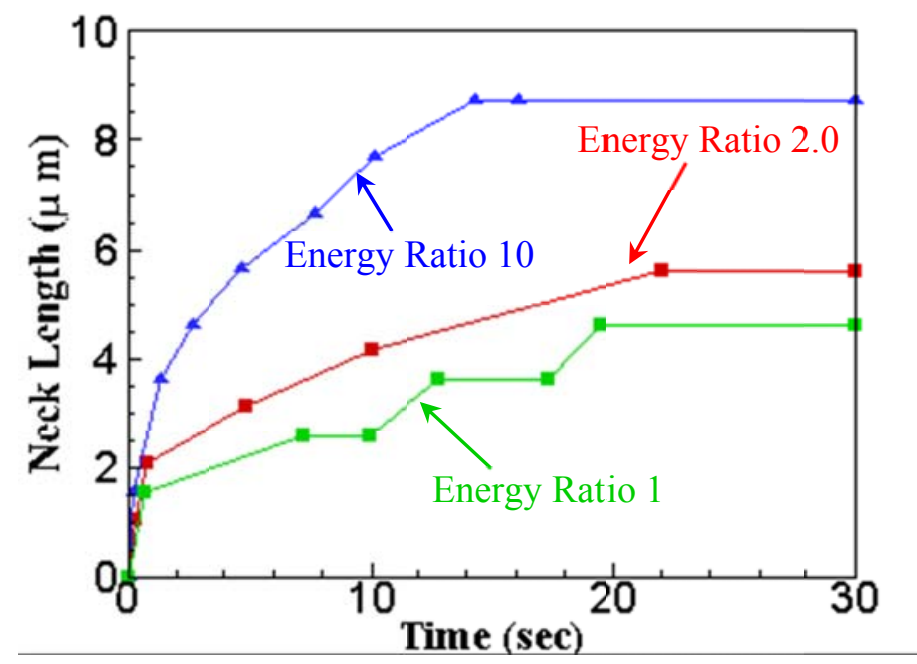

Figure 6. Neck length variation corresponding to different energy ratios

As explained in Eq. (41), the energy ratio $\left(\gamma_{\mathrm{s}} / \gamma_{\mathrm{gb}}\right)$ is related to the dihedral angle $(\varphi)$ of the grains which determines the equilibrium shape of the particles. Higher energy ratios correspond to higher dihedral angles which eventually governs the final neck length and grain configuration. Exponent values corresponding to energy ratio 10 is 5.4 , which indicates that the active diffusion mechanisms are surface and grain boundary diffusion which results in grain compaction during sintering. 


\subsection{Grain Growth:}

The governing densification mechanism - such as diffusion, rigid body motion, grain growth - is determined by the parameters used for simulation. The co-efficients $A, B$ and the interfacial properties play an important role in this case. Fig. (7) shows the grain growth in case of two unequal size particles.

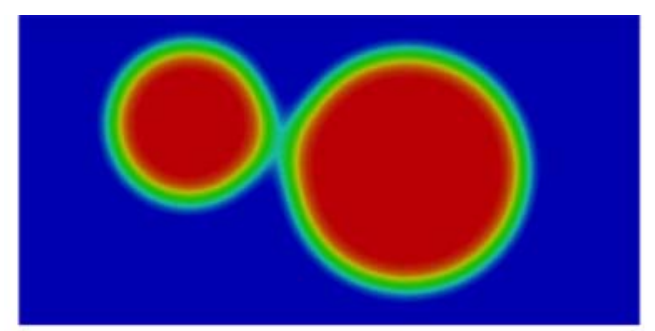

(a)

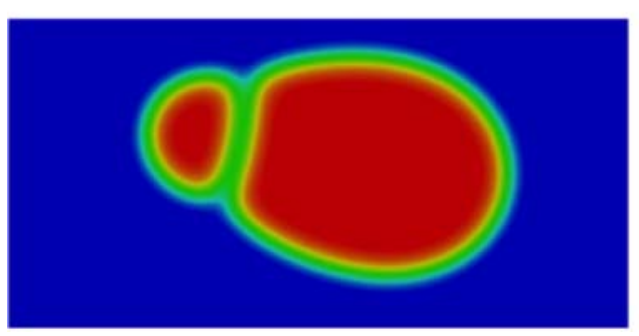

(c)

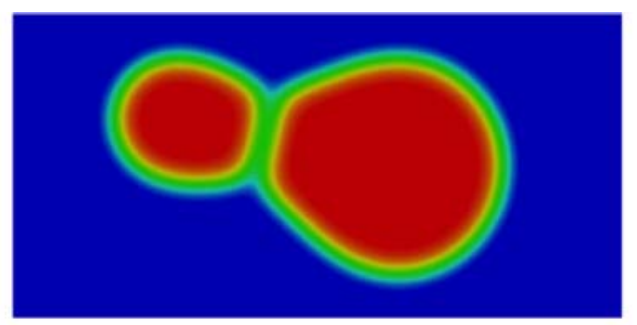

(b)

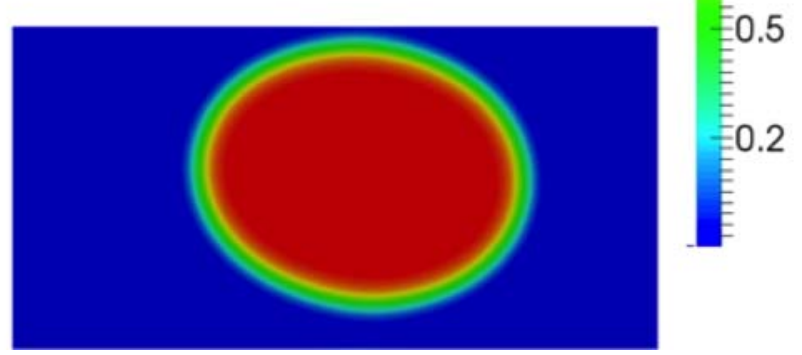

(d)

Figure 7. Grain Growth for model with two unequal size particles at a) $0 \mathrm{sec}$ b) $10 \mathrm{sec}$ c) $50 \mathrm{sec}$ d)100 sec

Variation of average grain size as a function of energy ratio and time is shown in Fig. (8). As observed in Fig. (8), as energy ration increases, rate of grain growth also increases. Higher grain growth rate indicates that the densification is governed by volumetric bulk diffusion. 


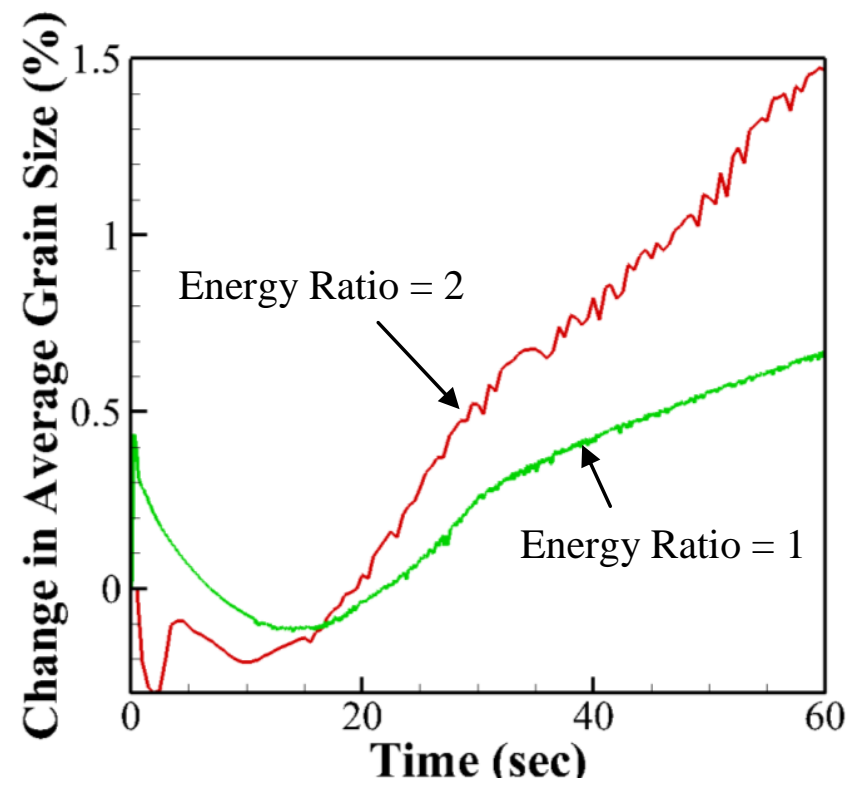

Figure 8. Effect of energy ratio on grain growth rate of particles during sintering

Grain growth is quantified as,

$$
D^{n}(t)-D_{0}^{n}=k t,
$$

where, $D(t)$ is average grain size at time $t, D_{0}$ is the average initial grain size and $k$ is rate constant. The value of the growth exponent is dictated by the predominant diffusion mechanism. Theoretical correlation between neck growth and grain growth exponents with the densification mechanism is provided in Table 3.

Table 5. Correlation of exponents to the densification mechanisms [50]

\begin{tabular}{|l|l|l|l|}
\hline $\begin{array}{l}\text { Neck Growth } \\
\text { Exponent }\end{array}$ & Sintering Mechanism & $\begin{array}{l}\text { Grain Growth } \\
\text { Exponent }\end{array}$ & Sintering Mechanism \\
\hline 5 & Volume Diffusion & 4 & Volume Diffusion \\
\hline 6 & GB Diffusion & 5 & GB/Surface Diffusion \\
\hline 7 & Surface Diffusion & & \\
\hline
\end{tabular}

Fig. (9) pictorially represents all the diffusion mechanisms during sintering, 


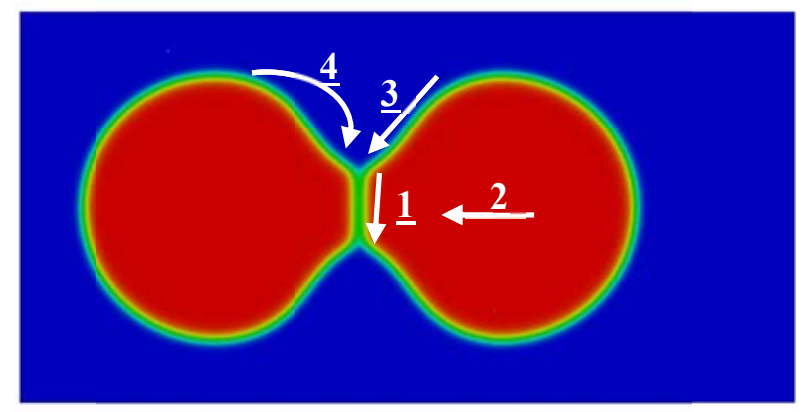

1. GB Diffusion

2. Volume diffusion

3. Surface Diffusion

4. Vaporization /

Vacancy Diffusion

\section{Figure 9. Diffusion paths along with diffusion mechanisms}

For the case with energy ratio 1 , the grain growth exponent was found to be 4 , which indicates the dominant densification mechanism is surface diffusion. Prediction from both neck growth and grain growth examination, point to the same densification mechanism at initial stage of sintering, i.e., surface diffusion. The effect of initial grain size and grain size distribution on the overall grain growth is shown in Fig. (10).

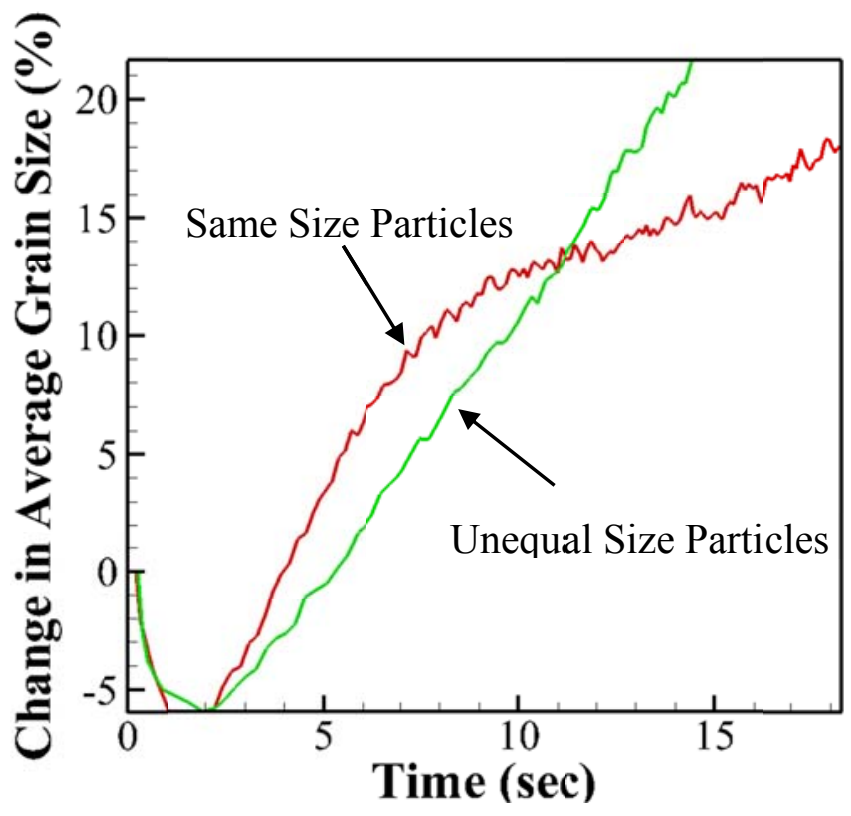

Figure 10. Effect of grain size distribution on the grain growth 
For the same size particles rate of increase in average grain size is higher at initial stage as compared to that of unequal size particle distribution case. However, at later stages as equilibrium is achieved grain size stabilizes. In the case of unequal particle size, larger particles absorbs the smaller one due to bulk diffusion and grain growth continues even at the later stage. Even though grain growth rate is slower, final average grain size is higher for unequal grain distribution. As observed, for equal size particles, at initial stage individual grain increases in size until it reaches adjacent grains. Once grains start interacting with each other, grain boundary diffuses and the rate of grain growth reduces. Initial negative change in grain size indicates individual grain shrinkage happening before any mass diffusion starts. In the case of unequal grain size distribution, smaller grains merge into larger grains. Therefore, some grains become larger and some grains reduce in size and eventually disappear. It order to restrain grain growth during sintering, it is advisable to use powder particles with approximately same average size.

\subsection{Evolution of Multiparticles:}

Current simulation is extended further to multi particle sintering. The simulations, here, start with microstructure of a powder materials having multiple circular particles and ends with the final polycrystalline configuration of the material. The microstructural images at different time steps are presented in Fig. (11). 


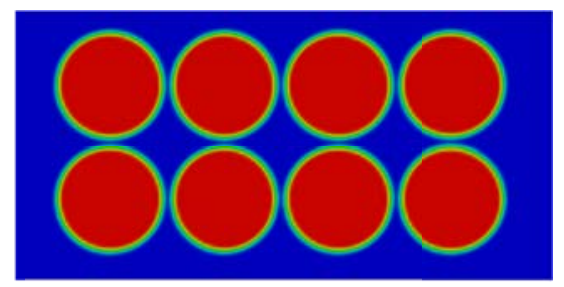

(a)

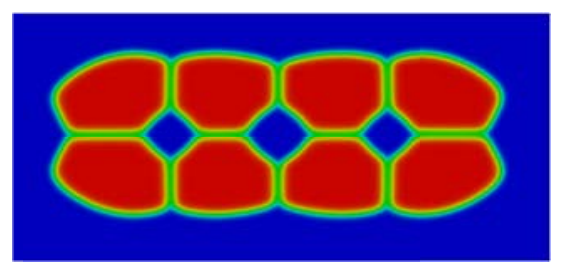

(d)

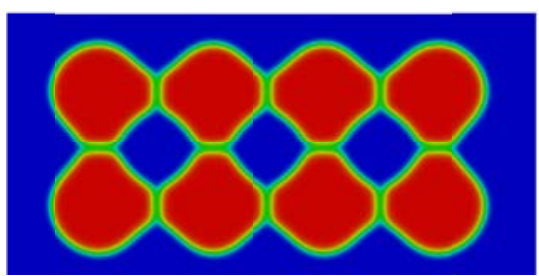

(b)

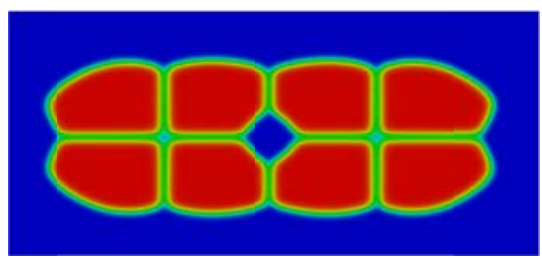

(e)

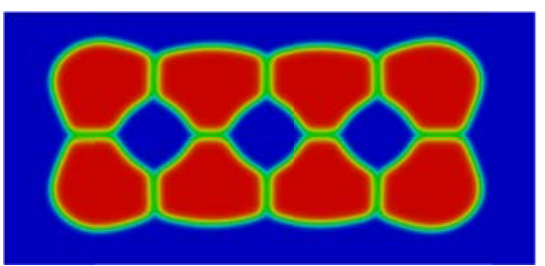

(c)

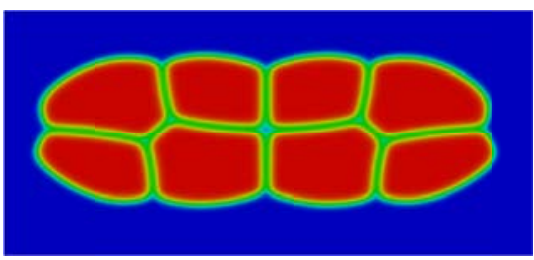

(f)

Figure 11. . Microstructural evolution of multiparticles during sintering process at time a) 0 sec b) $2 \mathrm{sec}$ c) $20 \mathrm{sec} \mathrm{d)} 100 \mathrm{sec}$ e) $125 \mathrm{sec}$ f) $156 \mathrm{sec}$

Fig. (11) depicts the temporal evolution of particles during sintering process. The particle compaction in this case is governed by the surface and grain boundary diffusion. Porosity of the material decreases over time. Depending on a grain's position and interaction with adjacent grains, diffusion behavior of each grain varies significantly. Surface diffusion of the grains on the edge (having pores on one side ) are higher than the intermediate grains (which has pores on both sides). So the influence of the grain diffusion on the pores are different at different positions which leads to closure of some pores earlier than others. Deformation \& shape change of adjacent grains effects the size reduction and closure time of a pore. The final density of the compacted structure improves with higher applied loading. For the current model, only small strain elastic deformation has been considered. Hence, particle density changes linearly over time until it reaches a maximum value. Changes in density are proportional to applied maximum pressure and deformation. Density change in this case is governed by the reduction in overall volume. This is in agreement with what Dahl [51] observed during sintering of yttria stabilized zirconium using a different sintering technique. 


\section{§4. Conclusion and Future Work:}

In the current work, the conventional phase field modeling approach with elastic loading and modified Cahn-Hilliard and Allen-Cahn equations for the advection terms are combined for modeling microstructure evolution during sintering. The model predicts the densification mechanisms during sintering through neck formation and grain growth. Consideration of elastic loading and rigid body motion leads to particle rotation and shape change. This explains the reason for obtaining a dense polycrystalline structure from powder with circular particles. It is observed that energy ratios play an important role in consolidation kinetics of the powder particles. Densification occurs faster as the energy ratio increases. It also influences the final density of the consolidated microstructure. Initial grain size and grain size distribution impact the grain growth and the densification mechanisms. Powder with equal size particles reduces the probability of grain growth.

Overall, the layout of a generic phase field study for modeling sintering based material processing is presented here. The prevailing densification mechanism considered here is diffusion. Our current model only takes into account the contribution of elastic loading. For further studies, it can be extended to incorporate plastic deformation of the material. Consideration of plasticity will activate other densification mechanisms enabling the study of the effect of maximum load and loading rate. This model can be applied to specific materials by incorporating appropriate diffusion and mobility parameters. Temperature sources can also be modeled to study effect of temperature.

\section{Acknowledgements}

We acknowledge the funding received from DoE-NETL supporting this research work. We are grateful to the MOOSE developers and the user community, for helping with technical discussions to overcome modeling hurdles. 


\section{References}

1. Olevsky, E. and L. Froyen, Constitutive modeling of spark-plasma sintering of conductive materials. Scripta Materialia, 2006. 55(12): p. 1175-1178.

2. Guyot, P., et al., Hot pressing and spark plasma sintering of alumina: Discussion about an analytical modelling used for sintering mechanism determination. Scripta Materialia, 2014. 8485: p. 35-38.

3. Y.T. Keun, J.H.J.a.K.H.A., Computer simulation of ceramic sintering processes. Journal of Ceramic Processing Research, 2002. 3(3): p. 6.

4. Vanmeensel, K., et al., Modelling of the temperature distribution during field assisted sintering. Acta Materialia, 2005. 53(16): p. 4379-4388.

5. Langer, J., M.J. Hoffmann, and O. Guillon, Direct comparison between hot pressing and electric field-assisted sintering of submicron alumina. Acta Materialia, 2009. 57(18): p. 5454-5465.

6. Muccillo, R. and E.N.S. Muccillo, An experimental setup for shrinkage evaluation during electric field-assisted flash sintering: Application to yttria-stabilized zirconia. Journal of the European Ceramic Society, 2013. 33(3): p. 515-520.

7. Tang, C.Y., et al., Crystallization Kinetics Analysis and SPS Consolidation of Amorphous Powders. Applied Mechanics and Materials, 2013. 477-478: p. 1259-1263.

8. A. Bruson, N.M., Consolidation Kinetics of Amorphous Powder under Warm pressing. Mater. Sci. Eng., 1984. 64: p. L13-L17.

9. Tokitani, M., Mechanism of Spark Plasma Sintering. Proceedings of the 2nd International meeting of Pacific Ceramic Societies, 1996.

10. Chaim, R., et al., Sintering and densification of nanocrystalline ceramic oxide powders: a review. Advances in Applied Ceramics, 2008. 107(3): p. 159-169.

11. Lee, D., et al., The effect of $H f C$ content on mechanical properties $H f C-W$ composites. International Journal of Refractory Metals and Hard Materials, 2014. 44: p. 49-53.

12. Chanthapan, S., et al., Sintering of tungsten powder with and without tungsten carbide additive by field assisted sintering technology. International Journal of Refractory Metals and Hard Materials, 2012. 31: p. 114-120.

13. Kim;, T.K., Effects of tungsten nanaoparticles on densification of tungsten powder. Rev. Edv. Mater. Sci., 2009. 21: p. 192-199.

14. Cha, S.I. and S.H. Hong, Microstructures of binderless tungsten carbides sintered by spark plasma sintering process. Materials Science and Engineering: A, 2003. 356(1-2): p. 381-389. 
15. J. R. Groza, J.D.C.a.M.K., Field Assisted sintering of nanocrystalline titanium nitride. J. Am. Ceramis Society, 2000. 83(5): p. 3.

16. Maizza, G., et al., Relation between microstructure, properties and spark plasma sintering (SPS) parameters of pure ultrafine WC powder. Science and Technology of Advanced Materials, 2007. 8(7-8): p. 644-654.

17. Grigoryev, E.G., Kinetics of Densification of powder materials under EPS. The Arabian Journal for Science and Engineering, 2009. 34: p. 29-33.

18. $\mathrm{Hu}, \mathrm{K}$., et al., Densification and microstructure evolution during SPS consolidation process in W-Ni-Fe system. Transactions of Nonferrous Metals Society of China, 2011. 21(3): p. 493-501.

19. Tikare, V., M. Braginsky, and E.A. Olevsky, Numerical Simulation of Solid-State Sintering: I, Sintering of Three Particles. Journal of the American Ceramic Society, 2003. 86(1): p. 49-53.

20. Tiwari, D., B. Basu, and K. Biswas, Simulation of thermal and electric field evolution during spark plasma sintering. Ceramics International, 2009. 35(2): p. 699-708.

21. Kraft, T. and H. Riedel, Numerical simulation of solid state sintering; model and application. Journal of the European Ceramic Society, 2004. 24(2): p. 345-361.

22. Y.T. Keun, J.H.J.a.K.H.A., Computer simulation of ceramic sintering processes. 2002.

23. Tonks, M.R., et al., An object-oriented finite element framework for multiphysics phase field simulations. Computational Materials Science, 2012. 51(1): p. 20-29.

24. Moelans, N., B. Blanpain, and P. Wollants, An introduction to phase-field modeling of microstructure evolution. Calphad, 2008. 32(2): p. 268-294.

25. Hu, S.Y. and C.H. Henager Jr, Phase-field simulation of void migration in a temperature gradient. Acta Materialia, 2010. 58(9): p. 3230-3237.

26. Grafe, U., et al., Simulations of the initial transient during directional solidification of multicomponent alloys using the phase field method. Modelling and Simulation in Materials Science and Engineering, 2000. 8(6): p. 871-879.

27. Loginova, I., G. Amberg, and J. Agren, Phase-field simulations of non-isothermal binary alloy solidification. Acta Materialia, 2001. 49(4): p. 573-581.

28. Uehara, T. and T. Tsujino, Phase field simulation of stress evolution during solidification. Journal of Crystal Growth, 2005. 275(1-2): p. e219-e224.

29. Jin, Y.M., A. Artemev, and A.G. Khachaturyan, Three-dimensional phase field model of lowsymmetry martensitic transformation in polycrystal: Simulation of zeta '(2) martensite in AuCd alloys. Acta Materialia, 2001. 49(12): p. 2309-2320. 
30. Yamanaka, A., T. Takaki, and Y. Tomita, Elastoplastic phase-field simulation of self-and plastic accommodations in martensitic transformation. Materials Science and Engineering: A, 2008. 491(1-2): p. 378-384.

31. Hu, S.Y. and L.Q. Chen, Solute segregation and coherent nucleation and growth near a dislocation - A phase-field model integrating defect and phase microstructures. Acta Materialia, 2001. 49(3): p. 463-472.

32. Rodney, D., Y. Le Bouar, and A. Finel, Phase field methods and dislocations. Acta Materialia, 2003. 51(1): p. 17-30.

33. Hu, S.Y., M.I. Baskes, and M. Stan, Phase-field modeling of microvoid evolution under elasticplastic deformation. Applied Physics Letters, 2007. 90(8).

34. Wang, Y. and J. Li, Phase field modeling of defects and deformation. Acta Materialia, 2010. 58(4): p. 1212-1235.

35. Kazaryan, A., et al., Generalized phase-field model for computer simulation of grain growth in anisotropic systems. Physical Review B, 2000. 61(21): p. 14275-14278.

36. Uehara, T., T. Tsujino, and N. Ohno, Elasto-plastic simulation of stress evolution during grain growth using a phase field model. Journal of Crystal Growth, 2007. 300(2): p. 530-537.

37. Aranson, I.S., V.A. Kalatsky, and V.M. Vinokur, Continuum field description of crack propagation. Physical Review Letters, 2000. 85(1): p. 118-121.

38. Karma, A., D.A. Kessler, and H. Levine, Phase-field model of mode III dynamic fracture. Physical Review Letters, 2001. 87(4).

39. Deng, J., A Phase Field Model of Sintering with Direction-Dependent Diffusion. Materials Transactions, 2012. 53(2): p. 385-389.

40. Prakash, C., et al., An analysis of the influence of grain boundary strength on microstructure dependent fracture in polycrystalline tungsten. International Journal of Fracture, 2016.

41. Wang, Y.U., Computer modeling and simulation of solid-state sintering: A phase field approach. Acta Materialia, 2006. 54(4): p. 953-961.

42. Cahn, J.W. and J.E. Hilliard, Free Energy of a Nonuniform System. I. Interfacial Free Energy. The Journal of Chemical Physics, 1958. 28(2): p. 258-267.

43. J. S. Lee, C.M., and Ch. Herzig, Grain Boundary Self-Diffusion in Polycrystalline Tungsten at Low Temperatures. phys. stat. sol., 1997: p. 202.

44. Mundy, J.N., et al., Self-diffusion in tungsten. Physical Review B, 1978. 18(12): p. 6566-6575.

45. Peterson, N.L., Diffusion in refractory metals. WAAD Technical report, 1960. 
46. Ahmed, K., et al., Phase field modeling of the effect of porosity on grain growth kinetics in polycrystalline ceramics. Modelling and Simulation in Materials Science and Engineering, 2013. 21(6): p. 065005.

47. M. Grujicic, H.Z., Atomistic Simulation of C3 (111) Grain Boundary Fracture in Tungsten Containing Various Impurities Int. J. of Refractory Metals \& Hard Materials 1997. 15: p. 14.

48. Zhang, L., et al., A quantitative comparison between and elements for solving the Cahn-Hilliard equation. Journal of Computational Physics, 2013. 236: p. 74-80.

49. Tonks, M.R., et al., Fully-coupled engineering and mesoscale simulations of thermal conductivity in UO 2 fuel using an implicit multiscale approach. Journal of Physics: Conference Series, 2009. 180(1): p. 012078.

50. Liu, L.L., et al., Phase-Field Simulation of Process in Sintering Ceramics. Advanced Materials Research, 2010. 154-155: p. 1674-1679.

51. Dahl, P., et al., Densification and properties of zirconia prepared by three different sintering techniques. Ceramics International, 2007. 33(8): p. 1603-1610. 